\title{
HOXA7 stimulates human hepatocellular carcinoma proliferation through cyclin E1/CDK2
}

\author{
YUEHUI LI ${ }^{1}$, XIAO HUI YANG ${ }^{2}$, SHU JUANG FANG ${ }^{1}$, CHANG FEI QIN $^{1}$, RUI LI SUN ${ }^{1}$, \\ ZHAO YANG LIU ${ }^{1}$, BIN YUAN JIANG ${ }^{1}$, XIANG WU ${ }^{1}$ and GUANCHENG LI ${ }^{1}$ \\ ${ }^{1}$ Tumor Immunobiology Laboratory of the Cancer Research Institute, Key Laboratory of Carcinogenesis and Cancer Invasion, \\ Ministry of Education, Key Laboratory of Carcinogenesis, Ministry of Health, Central South University, Changsha, Hunan; \\ ${ }^{2}$ Xiangya Third Hospital, Central South University, Changsha, Hunan, P.R. China
}

Received November 4, 2014; Accepted December 8, 2014

DOI: $10.3892 /$ or.2014.3668

\begin{abstract}
HOX genes are transcription factors that control morphogenesis, organogenesis and differentiation. Increasing evidence suggests that HOX genes play a role in hepatocellular carcinoma (HCC) progression; however few studies have defined the functional roles and mechanisms of action. In the present study, we used siRNA and forced-expression in multiple cell lines to define the role of HOXA7 in the regulation of proliferation of $\mathrm{HCC}$ in vitro and in vivo. Knockdown of endogenous HOXA7 decreased the proliferation of HepG2 and QGY-7703 cells. These changes were not associated with significant changes in cyclin D1 and CDK4. However, downregulation of HOXA7 significantly reduced cyclin E1 and CDK2 protein levels. Conversely, overexpression of HOXA7 in QSG-7701 cells stimulated proliferation and increased cyclin E1 and CDK2 protein levels. Our results confirmed that HOXA7 promoted cell proliferation, and these changes were mediated by cyclin E1/CDK2. These observations contribute to our understanding of the important roles of HOXA7 in HCC development and progression and HOXA7 could be a promising molecular target for the development of new diagnostic and therapeutic strategies for $\mathrm{HCC}$.
\end{abstract}

\section{Introduction}

Hepatocellular carcinoma (HCC) is the most common primary liver cancer and one of the most common malignancies in the world, accounting for approximately one million deaths per year (1). Although liver resection and local ablation are regarded as potentially curative treatment (2), its prognosis is poor. Most of the patients are diagnosed with advanced disease at presentation for which palliative therapy forms the mainstay

Correspondence to: Professor Guancheng Li, Tumor Immunobiology Laboratory of the Cancer Research Institute, Central South University, 110 Xiangya Road, Changsha, Hunan 410078, P.R. China E-mail: libsun@163.com

Key words: hepatocellular carcinoma, HOXA7, proliferation, siRNA, cyclin E1/CDK2 of treatment (3). To improve this situation, the development of novel molecular therapies against effective targets is an urgent issue.

Toward this direction, we previously cloned a novel tumor associated gene HTA (4). To further elucidate its mechanism in hepatoma carcinogenesis and its potential role as a cancer biomarker, research was carried out. The results showed that knockdown of endogenous HTA expression in the malignant hepatic cell line HepG2 (HepG2/si-HTA) by small interfering RNA (siRNA), attenuated cell growth, weakened its tumor formation ability in nude mice (5) while overexpression of the HTA gene in the hepatic cell line QSG-7701 (QSG-7701/HTA) via stable transfection promoted its proliferation rate, colony forming ability and altered the cell cycle distribution of the cell lines (6). Furthermore, in a previously study, a cDNA microarray analysis identified that many genes were differentially expressed in HepG2/si-HTA and QSG-7701/HTA cells. Following the knockdown and overexpression of HTA, the expression level of HOXA7 was changed (Li et al, unpublished data), which suggests that HTA and HOXA7 may be important in HCC development and progression.

Mammals have 39 HOX genes split between four groups (A-D) of linked genes on different chromosomes, which are thought to have arisen from a series of duplication events (7). HOX genes encode morphoregulatory transcription factors that specify positional identity during development and regulate differentiation, motility, adhesion and proliferation in adult tissues (8). Accumulating evidence suggests that aberrantly expressed HOX genes may play important roles in the biology of a variety of solid tumors and hematological malignancies (9-11). Although numerous studies have documented differences in HOX gene expression between normal and neoplastic tissues, relatively few studies have defined specific functional roles and mechanisms of action for HOX genes in human HCC.

The expression of several HOX genes was found to be altered in human HCC compared to that in normal tissue $(12,13)$. Overexpression of HOXA13 in human HCC cells was associated with the poorest prognosis of hepatocarcinomas (14). Cell biological approach demonstrated that misexpression of some HOX genes altered the malignant potential of human tumor cells in culture. Enforced 
expression of HOXA1 in human breast cancer cells resulted in enhancement of cell proliferation (15). Knockdown of HOXC10 expression by shRNA decreased the invasiveness of cervical cancer cells (16). Overexpression of HOXB7 in immortalized ovarian surface epithelial cells was found to enhance cellular proliferation (17), whereas overexpression of antisense to HOXB7 and HOXB13 suppressed Transwell invasion of SKOV-3 ovarian cancer cells (18). Forced overexpression of HOXD3 enhanced the invasive and metastatic abilities of lung cancer cells through the activation of integrin $\alpha v \beta 3$ and TGF- $\beta$ pathways (19-21).Thus, accumulating evidence suggests that HOX genes are involved in oncogenesis and malignant progression.

In the present study, through in vitro and in vivo expriments we investigated the role of HOXA7 in the regulation of proliferation in human HCC. The results revealed an important role for HOXA7 in promoting human HCC progression, mediated by cyclin E1 and CDK2.

\section{Materials and methods}

Cell culture. HCC cell lines, HepG2, Hep3B-2 and QGY-7703, and normal liver cell line, QSG-7701, were maintained in our laboratory and cultured in RPMI-1640 medium supplemented with $10 \%$ fetal bovine serum (FBS) (both from Gibco). Cells were maintained at $37^{\circ} \mathrm{C}$ in an atmosphere of humidified air with $5 \% \mathrm{CO}_{2}$.

Reverse transcription-PCR ( $R T-P C R)$. RNA isolated from the cells was reverse-transcribed and amplified using the One-Step RT-PCR System (Fermentas, Vilnius, Lithuania). The sets of primers for HOXA7 were: sense, 5'-CTTATACAATGTCAA CAGCC-3' and antisense, 5'-TCCTTATGCTCTTTCTTCC-3', 414 bp; sense, 5'-AATCCCATCACCATCTTCCA-3' and antisense, 5'-CCTGCTTCACCACCTTCTTG-3' for GAPDH, $580 \mathrm{bp}$. After heating at $95^{\circ} \mathrm{C}$ for $1 \mathrm{~min}$, the samples were exposed to 30 cycles (GAPDH, 25 cycles) of $95^{\circ} \mathrm{C}$ for $30 \mathrm{sec}$, $56^{\circ} \mathrm{C}$ for $30 \mathrm{sec}$ and $72^{\circ} \mathrm{C}$ for $1 \mathrm{~min} 30 \mathrm{sec}$, with a final extension at $72^{\circ} \mathrm{C}$ for $10 \mathrm{~min}$. Reaction products were separated on $1 \%$ agarose gels containing ethidium bromide, and the level of amplification was analyzed using a Phosphor Imager.

Western blot analysis. The cells were washed with cold phosphate-buffered saline (PBS) and lysed in Laemmli buffer (62.5 mM Tris-HCl, pH 6.8, 2\% SDS, 10\% glycerol, $50 \mathrm{mM}$ dithiothreitol and $0.01 \%$ bromophenol blue) for $10 \mathrm{~min}$ at $100^{\circ} \mathrm{C}$. Cell lysates were analyzed by SDS/PAGE and transferred electrophoretically to a polyvinylidene difluoride membrane. The blots were probed with specific antibodies by a secondary detection step. The immunoreactive proteins were revealed by an ECL kit. Western blotting was carried out using the following antibodies: rabbit anti-HOXA7 antibody (Santa Cruz Biotechnology), rabbit anti-cyclin D1 antibody (Cell Signaling Biotechnology), rabbit anti-cyclin E1 antibody (Abcam Biotechnology), rabbit anti-CDK4 antibody, and rabbit anti-CDK2 antibody (both from Santa Cruz Biotechnology).

Knockdown and overexpression of HOXA7 and vector construction. To knock down HOXA7 expression, we used the GV102-U6/Neo/GFP vector encoding a small hairpin
RNA directed against the target gene in the HepG2 and QGY-7703 cells. The target sequences for HOXA7 were 5'-TCGCCCACACGCTCTGTTT-3'. We used a negative universal control as a negative control (NC).

For transfection of the plasmid expression vector encoding human HOXA7, the DNA sequencing containing the full-length HOXA7 (917 bp) open reading frame flanked by BamHI (sense) and EcoRI (antisense) restriction sites was PCR amplified from the HepG2 cells. The primer sequences used were sense, 5'-TCATTCCTCCTCGTCC-3' and antisense, 5'-ATGAGTTCTTCGTATTATGAACG-3'. The resulting fragment was inserted into pcDNA3.1(+) to generate pcDNA3.1-HOXA7. The desired sequence was confirmed by direct DNA sequencing.

Cell transfection. For transfection, the $\mathrm{HCC}$ and normal liver cell lines were grown to $70 \%$ confluency and transfected in serum-free medium for $6 \mathrm{~h}$ with FuGENE6 (Roche) and the vector. After $48 \mathrm{~h}$, the cells were subjected to G418 selection $(600 \mu \mathrm{g} / \mathrm{ml})$, followed by limited dilution in 96 -well plates for the generation of individual cell clones. The cells were harvested 15 days later to analyze the expression of HOXA7 by RT-PCR using the primers listed above and by western blotting using the rabbit antibodies listed above.

MTT analysis. For the MTT analysis, cells were seeded with RPMI-1640 medium with $1 \%$ FBS at a density of $10^{3}$ cells/well in 96-well plates $(n=6)$, grown overnight, washed in PBS and incubated in RPMI-1640 with $10 \% \mathrm{FBS}$ at $37^{\circ} \mathrm{C}$ in $5 \% \mathrm{CO}_{2}$ for varying periods and exposed to fresh media every other day. During the last $4 \mathrm{~h}$ of each day of culture, the cells were treated with methyl thiazolyl tetrazolium (MTT; $50 \mu \mathrm{g} /$ well; Sigma, USA). The generated formazan was dissolved in dimethyl sulfoxide (DMSO) and measured as OD (490 nm) for detecting the cell viability.

Colony formation analysis. For the colony formation analysis, the cells at 1,000 cells/well in 6-cm plates were incubated with RPMI-1640 medium with 1\% FBS for $24 \mathrm{~h}$, and then cultured in RPMI-1640 medium with $10 \% \mathrm{FBS}$ at $37^{\circ} \mathrm{C}$ at $5 \% \mathrm{CO}_{2}$ for 2 weeks. The cell colonies were then washed twice with PBS, fixed with $4 \%$ paraformaldehyde for $15 \mathrm{~min}$ and stained with Giemsa for $30 \mathrm{~min}$. Individual clones consisting of $>50$ cells were counted. Clone forming efficiency for each individual type of cells was calculated, according to the number of colonies/the number of inoculated cells x $100 \%$.

Flow cytometric analysis. For the flow cytometric analysis, the cells were incubated with RPMI-1640 medium with 1\% FBS for $24 \mathrm{~h}$, and then cultured in RPMI-1640 medium with $10 \%$ FBS for $24 \mathrm{~h}(\mathrm{n}=3)$. The cells were harvested and resuspended in fixation fluid at a density of $10^{6} / \mathrm{ml}$. Propidium iodide (PI) $(1,500 \mu \mathrm{l})$ solution was added, and the cell cycle was detected by FACSCalibur (Becton-Dickinson).

Tumor formation in the nude mice. To test the influence of HOXA7 on the development of HCC, the tumor formation in nude mice was examined. Briefly, HepG2/si-HOXA7, HepG2/NC, QGY-7703/si-HOXA7 and QGY-7703/NC cells $\left(5 \times 10^{6}\right)$ were injected subcutaneously into 4 -week-old BALB/c 
A

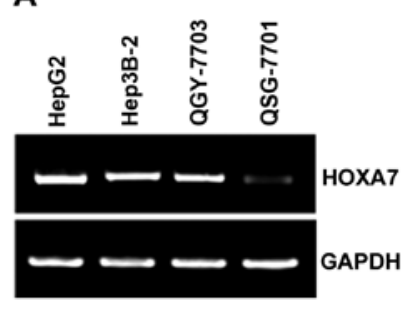

B

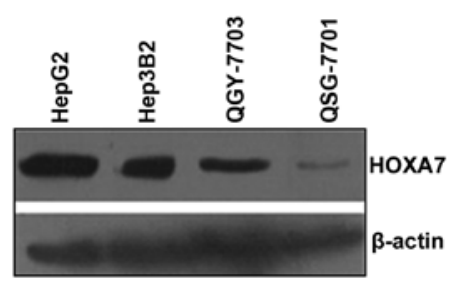

Figure 1. Expression of HOXA7 in the HepG2, Hep3B-2, QGY-7703 and QSG-7701 cell lines. (A) mRNA expression of HOXA7 and GAPDH detected by RT-PCR. (B) Protein expression of HOXA7 and $\beta$-actin as detected by western blotting.

nude mice ( $\mathrm{n}=4$ /group; Shanghai Laboratory Animal Center, Shanghai, China). The experimental pairs (HepG2/si-HOXA7, HepG2/NC, QGY-7703/si-HOXA7 and QGY-7703/NC) were carried out in the different mice. The development and growth of solid tumors were monitored by measuring the tumor size using a vernier caliper in a blinded manner every 5 days for a 40-day period: Tumor volume $=$ width $^{2} \mathrm{x}$ length $\mathrm{x} 0.5$. At the

end of the experiment, all mice were sacrificed, and individual tumor weights were measured.

Statistical analysis. All data are expressed as means \pm standard deviation. Difference among groups were determined by ANOVA, and the comparison between two groups was analyzed by the Student's t-test using GraphPad Prism software version 4.0 (GraphPad Software, Inc., San Diego, CA, USA). A probability value of $\mathrm{P}<0.05$ was considered to indicate a statistically significant result.

\section{Results}

mRNA and protein expression of HOXA7 in the HCC and normal liver cell lines. We assessed HOXA7 mRNA and protein expression in the HCC HepG2, Hep3B-2 and QGY-7703 cell lines and in the normal liver QSG-7701 cell line. The results of the semi-quantitative RT-PCR and western blotting showed that HOXA7 mRNA was detected in the HepG2, Hep3B-2, QGY-7703 and QSG-7701 cells, while HOXA7 protein was mainly expressed in the HepG2, Hep3B-2 and QGY-7703 cells (Fig. 1A and B).
A
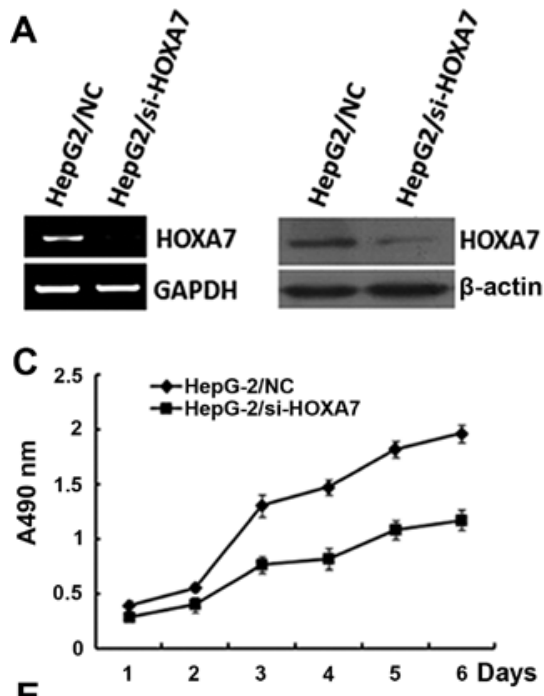

E

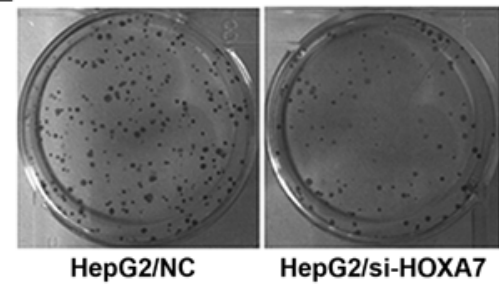

G

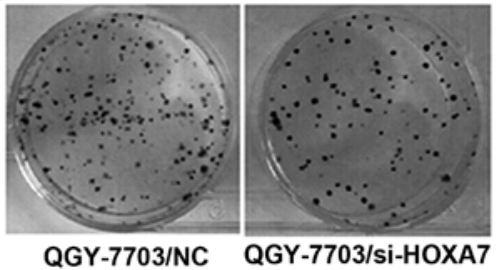

B
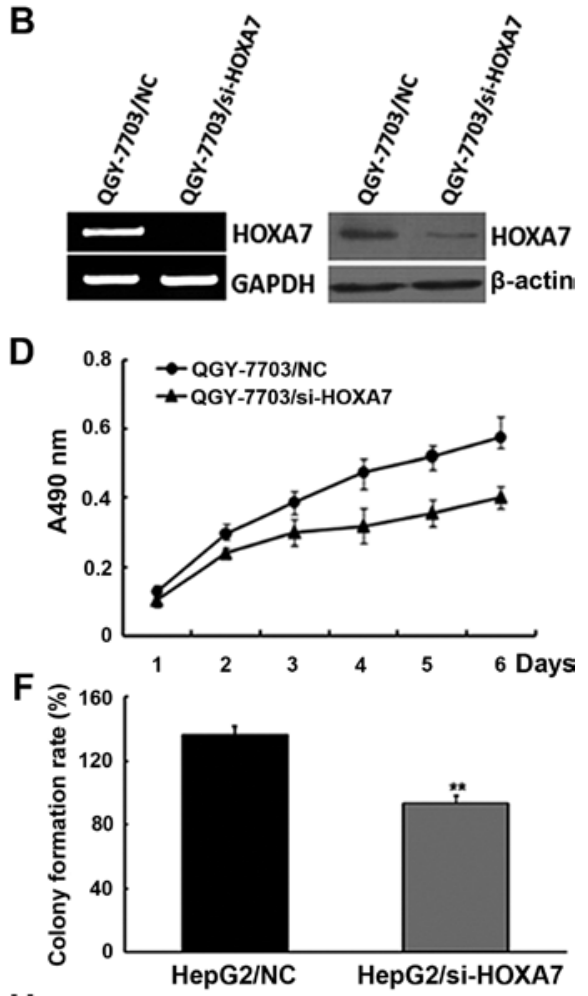

H

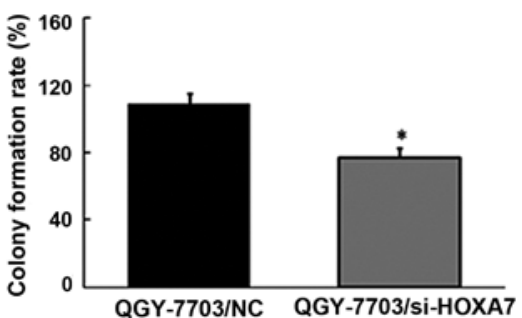

Figure 2. Impact of the knockdown of HOXA7 on the proliferation of HCC cells. (A and B) RT-PCR and western blotting verified the knockdown effect of HOXA7 in the HepG2 and QGY-7703 cell lines. (C and D) Cell proliferation curves of HepG2/si-HOXA7, QGY-7703/si-HOXA7 and negative control cells as detected by MTT assay. (E and F) Colony formation analysis of HepG2/si-HOXA7 and HepG2/NC cell lines ("P<0.01, HepG2/si-HOXA7 vs. HepG2/NC). ( $\mathrm{G}$ and $\mathrm{H}$ ) Colony formation analysis of QGY-7703/si-HOXA7 and QGY-7703/NC cell lines ("P<0.05, QGY-7703/si-HOXA7 vs. QGY-7703/NC). 
A
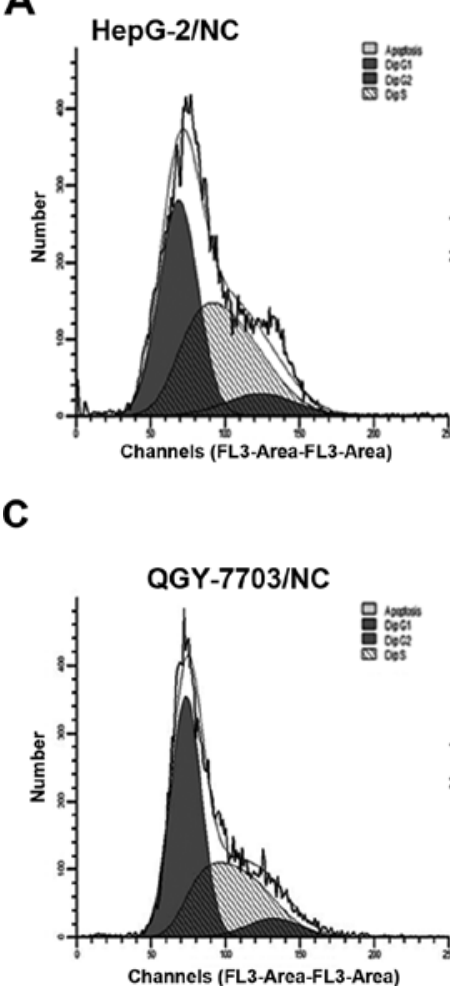

C

Channels (FL3-Area-FL3-Area)
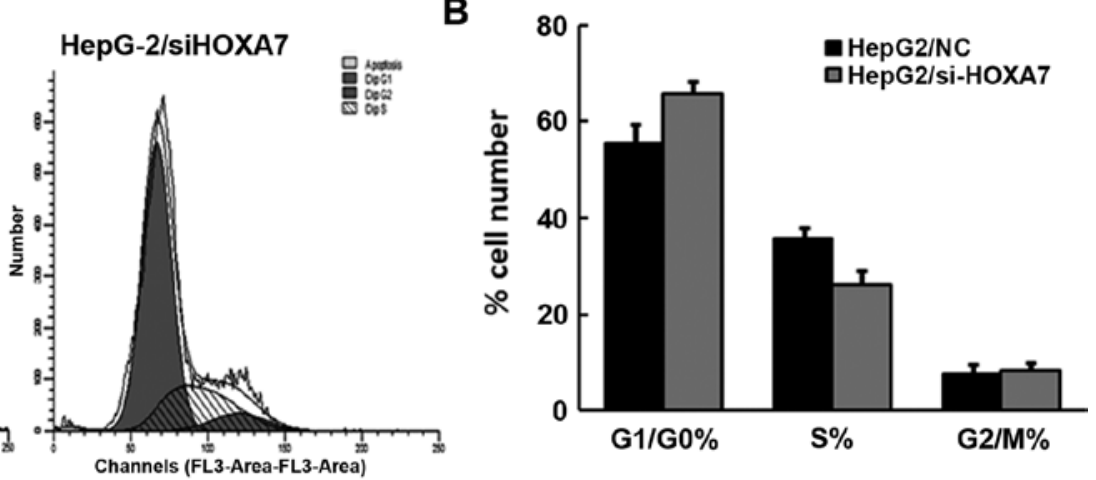
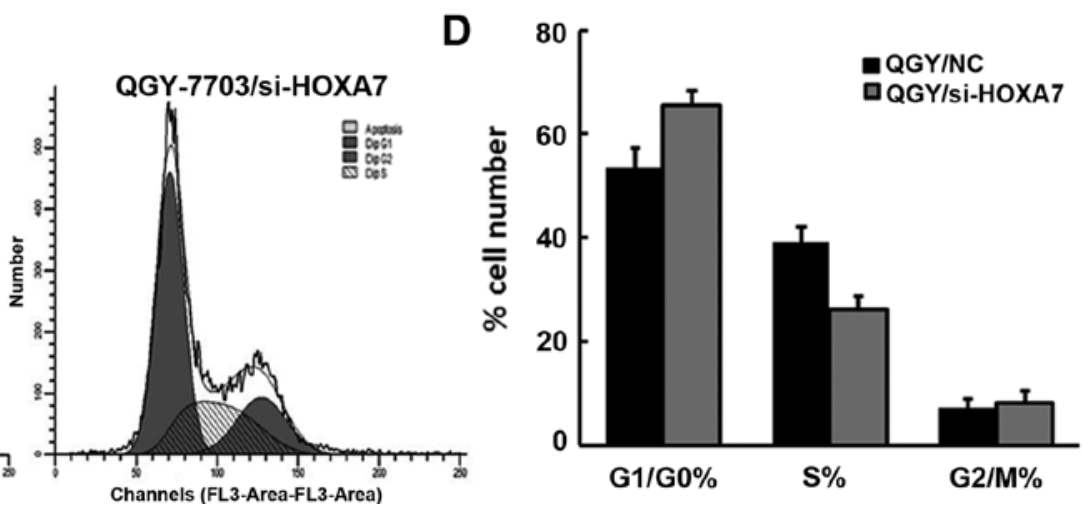

Figure 3. Cell cycle distribution. (A) Cell cycle distribution of HepG2/si-HOXA7 and HepG2/NC cells as detected by flow cytometry. (B) The percentage of HepG2/si-HOXA7 and HepG2/NC cells in the phases of the cell cycle. (C) Cell cycle distribution of QGY-7703/si-HOXA7 and QGY-7703/NC cells as detected by flow cytometry. (D) The percentage of QGY-7703/si-HOXA7 and QGY-7703/NC cells in the phases of the cell cycle.

Impact of the knockdown of HOXA7 on the proliferation of HCC cells. To study the biological function of HOXA7, we generated the RNAi vector containing siRNA specifically targeting HOXA7 to stably knock down the endogenous expression of HOXA7 in the HepG2 and QGY-7703 cells. As shown in Fig. 2A and B, compared to the control (HepG2/NC and QGY-7703/NC), cells transfected with si-HOXA7 had significantly decreased levels of HOXA7 mRNA and protein.

To study the impact of si-HOXA7 on the proliferation of HCC cells, we examined the proliferative efficiency of decreased HOXA7 in the HepG2 and QGY-7703 cells by MTT analysis. Following a 6-day period, the proliferation of the HepG2/si-HOXA7 and QGY-7703/si-HOXA7 cells was much slower than that of the HepG2/NC and QGY-7703/NC cells, and significantly high numbers of HepG2/NC and QGY-7703/NC cells were observed from day 3 (Fig. 2C and D). A similar pattern of inhibition following reduced HOXA7 expression in HepG2 and QGY-7703 cells was achieved in the colony formation assay (Fig. 2E-H). Therefore, the low proliferative activity and the low number of cell colonies in the HepG2/si-HOXA7 and QGY-7703/si-HOXA7 cells demonstrated that downregulation of HOXA7 expression inhibited cell proliferation in vitro.

Cell proliferation was also detected by flow cytometry. The results showed that HepG2 and QGY-7703 cells with si-HOXA7 exhibited cell cycle arrest in the G1 phase, which inhibited the proliferation of HepG2 and QGY-7703 cells (Fig. 3A-D). This result was consistent with the MTT analysis.

Overexpression of HOXA7 promotes QSG-7701 cell proliferation. Given that downregulation of HOXA7 inhib- ited HepG2 and QGY-7703 cell proliferation in vitro, we hypothesized that HOXA7 promotes QSG-7701 cell proliferation. To test this, QSG-7701 cells were transfected with a plasmid encoding HOXA7. Compared to the control cells (QSG-7701-C), cells transfected with the plasmid encoding HOXA7 (QSG-7701-HOXA7) had increased levels of HOXA7 mRNA and protein (Fig. 4A). MTT analysis showed that the proliferation rate of the QSG-7701-HOXA7 cells was much higher than that in the QSG-7701-C cells (Fig. 4B). Colony formation analysis revealed a larger number of cell colonies in the QSG-7701-HOXA7 cells. The results demonstrated that upregulation of HOXA7 expression promoted cell proliferation in vitro (Fig. $4 \mathrm{C}$ and D).

Cell proliferation was also detected by flow cytometry. The results showed that QSG-7701/HOXA7 cells underwent cell cy arrested the cell cycle in the G1 phase and an increased number of cells were in the $\mathrm{S}$ phase of the cell cycle, which promoted the proliferation of QSG-7701 cells (Fig. 4E and F). This result is in line with the above analysis.

Tumor formation in nude mice. Given that downregulation of HOXA7 inhibited HCC cell proliferation and upregulation of HOXA7 promoted HCC cell proliferation in vitro, we hypothesized that HOXA7 promotes HCC development in vivo. To further determine the role of HOXA7 in tumorigenesis and development of HCC, HepG2/si-HOXA7 and QGY-7703/si-HOXA7 or HepG2/NC and QGY-7703/NC cells were injected subcutaneously into nude mice. The development of the tumors was monitored for 40 days. As shown in Fig. 5A and B, HOXA7-knockdown tumors emerged later 

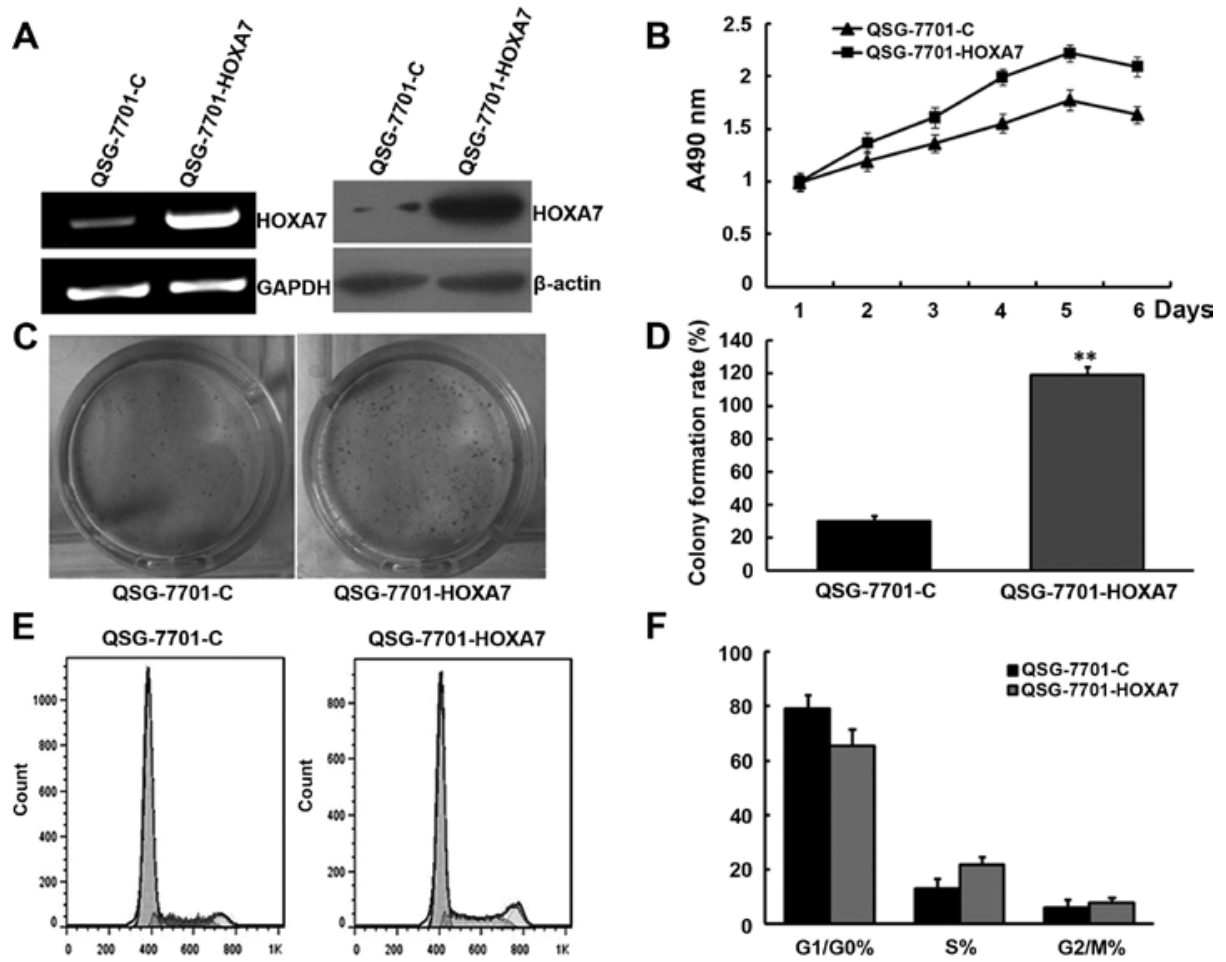

$\mathbf{F}$

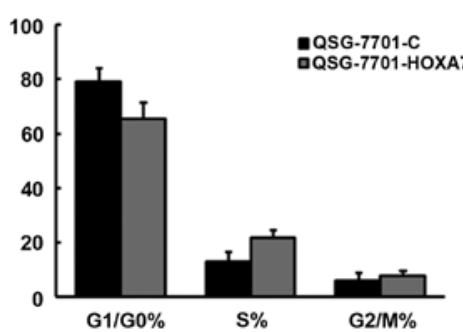

Figure 4. Overexpression of HOXA7 promotes QSG-7701 cell proliferation. (A) RT-PCR and western blotting verified the overexpression of HOXA7 in the QSG-7701 cells. (B) Cell proliferation curve of QSG-7701-HOXA7 and negative control cells as detected by MTT analysis. (C and D) Colony formation analysis of the QSG-7701-HOXA7 and QSG-7701-C cell lines ("P<0.01, QSG-7701-HOXA7 vs. QSG-7701-C). (E) Cell cycle distribution of QSG-7701-HOXA7 and QSG-7701-C cells as detected by flow cytometry. (F) The percentage of QSG-7701-HOXA7 and QSG-7701-C cells in each phase of the cell cycle.

A

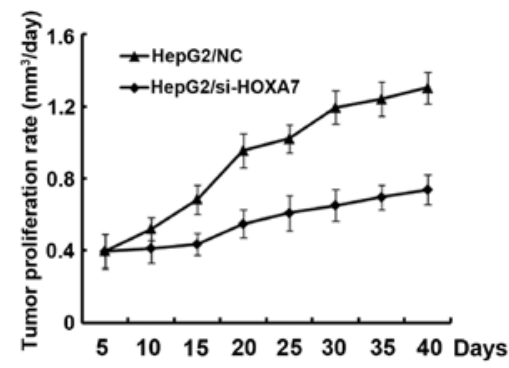

C

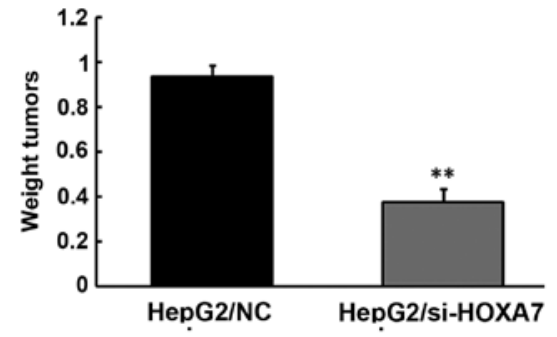

E

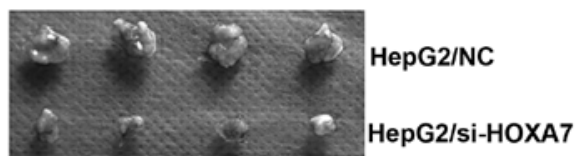

G

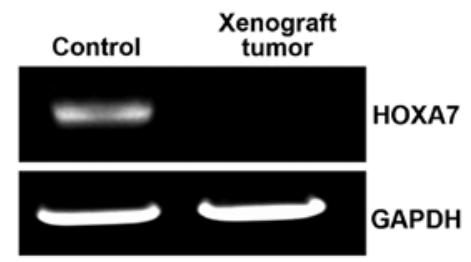

B

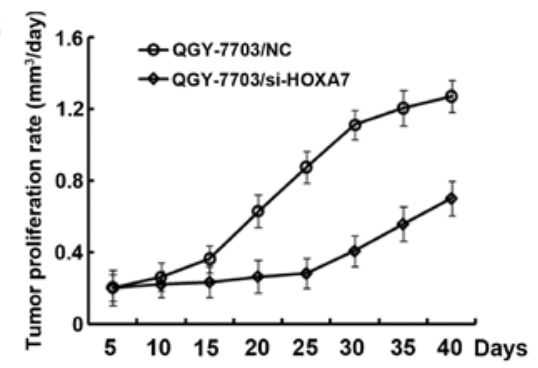

D

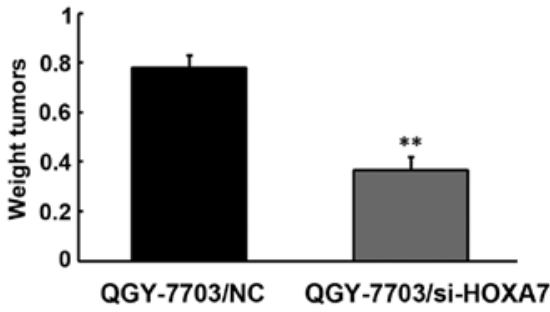

F

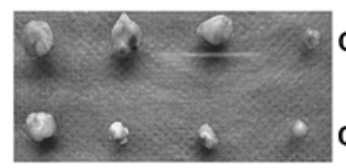

QGY-7703/NC

QGY-7703/si-HOXA7

$\mathbf{H}$

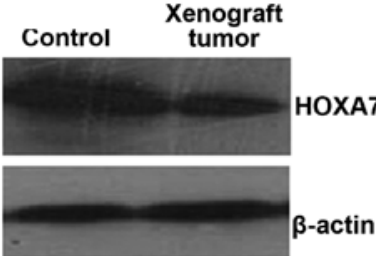

Figure 5. Tumor formation of the HepG2/si-HOXA7, QGY-7703/si-HOXA7 and control cell xenograft tumors in the Balb/c nude mice. (A and B) Tumor volume was measured every 5 days. (C-F) Forty days after tumor cell injection, the mice were sacrificed and the tumor weight was recorded. (G and H) RT-PCR and western blot analyses of the expression of HOXA7 in the representative xenograft tumors. 
A

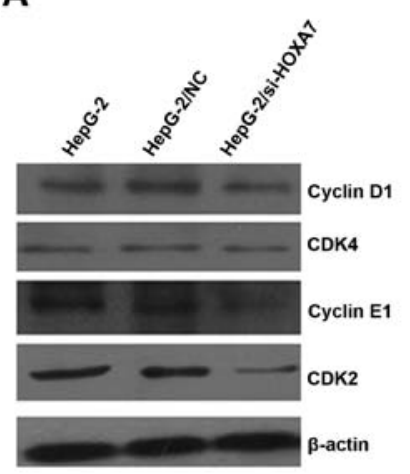

B

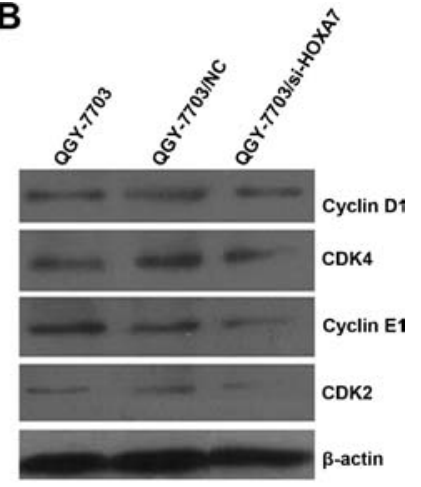

C

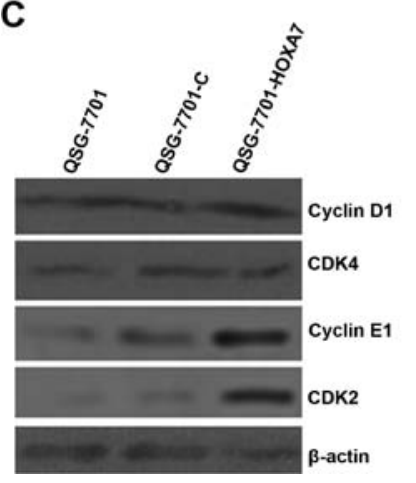

D

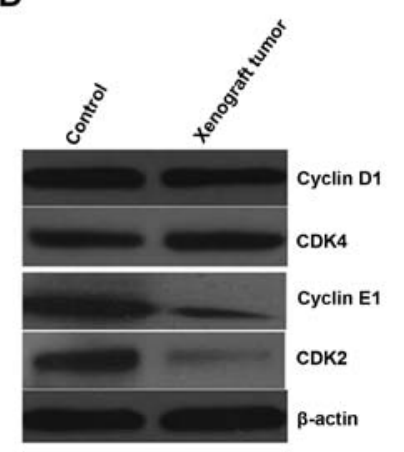

Figure 6. HOXA7 affects the expression of cell cycle-related proteins in the $\mathrm{HCC}$ and normal hepatic cells in the xenograft tumors. (A and B) Western blot analyses detected the expression of cyclin D1/CDK4 and cyclin E1 and CDK2 in the HCC cells. (C and D) Western blotting detected the expression of cyclin D1/CDK4 and cyclin E1 and CDK2 in normal hepatic cells and xenograft tumors.

and grew more slowly compared to the control tumors. At the end of the experimental period, the final weights of the HOXA7-knockdown tumors $(0.376 \pm 0.951$ and $0.368 \pm 0.093 \mathrm{~g})$ were found to be markedly lighter than the tumor weights in the controls $(0.936 \pm 0.98$ and $0.736 \pm 0.895$ g) (Fig. 5C-F). RT-PCR and western blotting of HOXA7 in the xenograft tumors indicated that increased HOXA7 expression had been maintained throughout the experimental time course (Fig. 5G and $\mathrm{H}$ ). Collectively, these data indicate that HOXA7 promoted xenograft tumor development in vivo.

HOXA7 affects the expression of cell cycle-related proteins in the HCC cells, normal hepatic cells and xenograft tumors. Cyclin D/CDK4 and cyclin E/CDK2 are important for cell cycle progression in metazoans and are frequently overexpressed in cancer cells. In view of the effects of HOXA7 on cell proliferation, we investigated whether knockdown of HOXA7 influences cyclin D1/CDK4 and cyclin E1/CDK2 expression in the HCC cells, normal hepatic cells and xenograft tumors. The total cellular levels of cyclin D1/CDK4, and cyclin E1/CDK2 were analyzed by western blotting. The results showed that HOXA7 had no conclusive effect on cyclin D1/CDK4 protein levels. However, downregulation of endogenous HOXA7 reduced cyclin E1/CDK2 protein levels (Fig. 6A and B). Moreover, western blotting also confirmed these results in the QSG-7701-HOXA7 cells and xenograft tumors (Fig. 6C and D).

\section{Discussion}

HOX genes are regulatory genes encoding nuclear proteins that act as transcription factors, which regulate aspects of morphogenesis and cell differentiation. There is accumulating evidence that HOX genes play important roles in oncogenesis, including ovarian, colon, lung and prostate cancer progression $(22,23)$. However, the specific role of the HOX genes in HCC cell function and oncogenesis has not been extensively explored. In the present study, we showed for the first time a significant role of HOXA7 in the regulation of HCC cell proliferation and cyclin E1/CDk2 expression. These findings indicate a new mechanism for HOX-mediated cell proliferation through the regulation of cyclin E1/CDK2 expression.

We first demonstrated that all three types of HCC cells express HOXA7. The expression of HOXA7 in the HCC cells indicated that HOXA7 exerted a potential role in cell regulation. In the present study, HepG-2 and QGY-7703 cells were used as an HCC tumor cell model, and QSG-7701 cells were used as a normal hepatic cell model. Quantitative analysis showed that the expression level of HOXA7 was significantly higher in the HCC tumor cells. It has been reported that many cancers, including leukemia, colon, skin, prostate, breast and ovarian cancers, have shown alterations in the expression patterns of HOX genes (24). The overexpression of HOX genes has been widely associated with a variety of carcinomas (25). Therefore, our results are consistent with previous observations that HOX genes are highly expressed in a variety of carcinomas. HOXA7, in particular, plays a role in HCC cell proliferation. In the present study, we performed siRNA-mediated knockdown of HOXA7 in a high HOXA7expressing $\mathrm{HCC}$ cell line. Conversely, we overexpressed HOXA7 in a low HOXA7-expressing QSG-7701 cell line to determine how HOXA7 regulates hepatic cell growth. The results showed that HOXA7 exerted a specific effect on HCC cell proliferation; knockdown of HOXA7 induced a decrease in $\mathrm{HCC}$ proliferation, while overexpression of HOXA7 in QSG-7701 cells significantly promoted cell growth. A previous study indicated that HOXA7 expression was increased during mitosis in cultured granulosa cells. Moreover, growth differentiation factor-9 (GDF-9), which enhances early follicular growth and differentiation, increased HOXA7 protein expression and regulated the expression of HOXA7 cofactors in granulosa cells $(26,27)$. These results support the hypothesis that HOXA7 modulates the growth and oncogenesis of human HCC cells.

Since cell proliferation is controlled by progression through the cell cycle, which is regulated by many proliferative signaling cascades, we carried out flow cytometric analysis to assess cell cycle distribution. The normal cell cycle follows ordinary steps, while cancer cells grow without regulation. The rate of progression in the cell cycle is decided by cyclins and cyclin-dependent kinases (CDKs). The entering of each phase is controlled by a specific cyclin-CDK complex. CDK is a member of the serine-threonine kinase family since a cyclin binds to a CDK and starts the phosphorylation of its serine and threonine site $(28,29)$. It has been reported that the G1-S checkpoint is mainly regulated by a series of cyclins and CDK, which are predominantly cyclin D/CDK4, CDK6, and 
cyclin $\mathrm{E} / \mathrm{CDK} 2$ (30). In HCC, cyclin E1 is an ideal candidate to be suppressed as an anti-HCC strategy as it is overexpressed in $~ 70 \%$ of HCC patients (31); overexpression was found to be directly correlated with HCC grade and a poor prognosis (32). The cyclin E-CDK2 complex guides the process from G1 to $\mathrm{S}$ phase, while the cyclin A-CDK2 complex is required to pass through the $\mathrm{S}$ phase $(33,34)$. In the present study, we showed that HOXA7 triggered cell cycle arrest in the G0/G1 phase. Cyclin E1/CDK2 is known to be involved in numerous cellular processes such as growth, motility and differentiation (35). The expression levels of cyclin E1/CDK2 have been correlated with the pathogenesis of a broad range of human types of cancers (36). In the present study, we examined the effect of cyclin E1/CDK2 on HCC cell growth. As observed in multiple cell types (37), cyclin E1/CDK2 activation was found to promote cell proliferation and stimulate the replication of cells. In the present study, the further identified cyclin E1/CDK2 as a novel downstream target of HOXA7.

In summary, our present data support a novel mechanistic role for the HOXA7 modulation of HCC cell proliferation via cyclin E1/CDK2. This finding indicates the effect of HOXA7 on HCC cell growth, motility and cellular proliferation, thus yielding new potential targets for the treatment of HCC tumors, and other cell proliferation dysfunctions of the cancers that are involved in the aberrant expression of HOX genes.

\section{Acknowledgements}

This study was supported by the National Natural Science Foundation of China (81201903), the Open-End Fund for the Valuable and Precision Instruments of Central South University (CSU2C2013048), Hunan, China.

\section{References}

1. Hao K, Luk JM, Lee NP, et al: Predicting prognosis in hepatocellular carcinoma after curative surgery with common clinicopathologic parameters. BMC Cancer 9: 389, 2009.

2. Song TJ, Ip EW and Fong Y: Hepatocellular carcinoma: current surgical management. Gastroenterology 127 (Suppl 1): S248-S260, 2004.

3. Paul SB, Gamanagatti SR, Mukund A, Abbas SZ and Acharya SK: Transarterial chemoembolization for hepatocellular carcinoma: significance of extrahepatic collateral supply. Indian J Cancer 48 339-344, 2011

4. Liu Y, Zhao Y, Fang S, Li Y and Li G: Molecular cloning and alternative splicing analysis of hepatoma associated gene HTA. Zhong Nan Da Xue Xue Bao Yi Xue Ban 38: 869-875, 2013 (In Chinese).

5. Liu Y, Li Y, Guo F, et al: Identification of HTA as a novel-specific marker for human hepatocellular carcinoma. J Cancer Res Clin Oncol 136: 1187-1192, 2010.

6. Liu Y, Zhao Y, Ju Q, et al: Molecular clone and functional study of a novel hepatoma associated gene. Int J Oncol 42: 1105-1112, 2013.

7. Scott MP: A rational nomenclature for vertebrate homeobox (HOX) genes. Nucleic Acids Res 21: 1687-1688, 1993.

8. Kawazoe Y, Sekimoto T, Araki M, Takagi K, Araki K and Yamamura K: Region-specific gastrointestinal Hox code during murine embryonal gut development. Dev Growth Differ 44: $77-84,2002$.

9. Abate-Shen C: Deregulated homeobox gene expression in cancer: cause or consequence? Nat Rev Cancer 2: 777-785, 2002.

10. Samuel S and Naora H: Homeobox gene expression in cancer: insights from developmental regulation and deregulation. Eur J Cancer 41: 2428-2437, 2005.

11. Argiropoulos B and Humphries RK: Hox genes in hematopoiesis and leukemogenesis. Oncogene 26: 6766-6776, 2007.
12. Kanai M, Hamada J, Takada M, et al: Aberrant expressions of $H O X$ genes in colorectal and hepatocellular carcinomas. Oncol Rep 23: 843-851, 2010.

13. Cillo C, Schiavo G, Cantile M, et al: The HOX gene network in hepatocellular carcinoma. Int J Cancer 129: 2577-2587, 2011.

14. Boyault S, Rickman DS, de Reynies A, et al: Transcriptome classification of HCC is related to gene alterations and to new therapeutic targets. Hepatology 45: 42-52, 2007.

15. Zhang X, Zhu T, Chen Y, Mertani HC, Lee KO and Lobie PE: Human growth hormone-regulated HOXA1 is a human mammary epithelial oncogene. J Biol Chem 278: 7580-7590, 2003.

16. Zhai Y, Kuick R, Nan B, et al: Gene expression analysis of preinvasive and invasive cervical squamous cell carcinomas identifies $\mathrm{HOXC10}$ as a key mediator of invasion. Cancer Res 67: 10163-10172, 2007

17. Naora H, Yang YQ, Montz FJ, Seidman JD, Kurman RJ and Roden RB: A serologically identified tumor antigen encoded by a homeobox gene promotes growth of ovarian epithelial cells. Proc Natl Acad Sci USA 98: 4060-4065, 2001.

18. Yamashita T, Tazawa S, Yawei Z, et al: Suppression of invasive characteristics by antisense introduction of overexpressed HOX genes in ovarian cancer cells. Int J Oncol 28: 931-938, 2006.

19. Hamada J, Omatsu T, Okada F, et al: Overexpression of homeobox gene HOXD3 induces coordinate expression of metastasis-related genes in human lung cancer cells. Int J Cancer 93: 516-525, 2001.

20. Miyazaki YJ, Hamada J, Tada M, et al: HOXD3 enhances motility and invasiveness through the TGF- $\beta$-dependent and -independent pathways in A549 cells. Oncogene 21: 798-808, 2002.

21. Ohta H, Hamada J, Tada M, et al: HOXD3-overexpression increases integrin $\alpha v \beta 3$ expression and deprives E-cadherin while it enhances cell motility in A549 cells. Clin Exp Metastasis 23: 381-390, 2006.

22. Grier DG, Thompson A, Kwasniewska A, McGonigle GJ, Halliday HL and Lappin TR: The pathophysiology of HOX genes and their role in cancer. J Pathol 205: 154-171, 2005.

23. Bhatlekar S, Fields JZ and Boman BM: HOX genes and their role in the development of human cancers. J Mol Med 92: 811-823, 2014.

24. Zhang Y, Huang Q, Cheng JC, et al: Homeobox A7 increases cell proliferation by up-regulation of epidermal growth factor receptor expression in human granulosa cells. Reprod Biol Endocrinol 8: 61, 2010.

25. Nunes FD, de Almeida FC, Tucci R and de Sousa SC: Homeobox genes: a molecular link between development and cancer. Pesqui Odontol Bras 17: 94-98, 2003.

26. Ota T, Choi KB, Gilks CB, Leung PC and Auersperg N: Cell type- and stage-specific changes in HOXA7 protein expression in human ovarian folliculogenesis: possible role of GDF-9. Differentiation 74: 1-10, 2006.

27. Ota T, Asahina H, Park SH, et al: HOX cofactors expression and regulation in the human ovary. Reprod Biol Endocrinol 6: 49, 2008.

28. Hartwell LH and Weinert TA: Checkpoints: controls that ensure the order of cell cycle events. Science 246: 629-634, 1989.

29. Kamb A, Gruis NA, Weaver-Feldhaus J, et al: A cell cycle regulator potentially involved in genesis of many tumor types. Science 264: 436-440, 1994.

30. Pines J: Protein kinases and cell cycle control. Semin Cell Biol 5: 399-408, 1994.

31. Jung YJ, Lee KH, Choi DW, et al: Reciprocal expressions of cyclin E and cyclin D1 in hepatocellular carcinoma. Cancer Lett 168: 57-63, 2001.

32. Masaki T, Shiratori Y, Rengifo W, et al: Cyclins and cyclin-dependent kinases: comparative study of hepatocellular carcinoma versus cirrhosis. Hepatology 37: 534-543, 2003.

33. Dehay C and Kennedy H: Cell-cycle control and cortical development. Nat Rev Neurosci 8: 438-450, 2007.

34. van den Heuvel S and Harlow E: Distinct roles for cyclin-dependent kinases in cell cycle control. Science 262: 2050-2054, 1993.

35. Liu QX, Wang XF, Ikeo K, Hirose S, Gehring WJ and Gojobori T: Evolutionarily conserved transcription factor Apontic controls the G1/S progression by inducing cyclin $E$ during eye development. Proc Natl Acad Sci USA 111: 9497-9502, 2014.

36. Gladden AB and Diehl JA: Cell cycle progression without cyclin E/CDK2: breaking down the walls of dogma. Cancer Cell 4: 160-162, 2003.

37. Rath SL and Senapati S: Why are the truncated cyclin Es more effective CDK2 activators than the full-length isoforms? Biochemistry 53: 4612-4624, 2014. 